\title{
Black chokeberry (Aronia melanocarpa) juice residue and its ethanol extract decrease serum lipid levels in high-fat diet-fed C57BL/6J mice
}

\author{
NANA MIKAMI ${ }^{1,2}$, YUKIE HOSOTANI ${ }^{1,3}$, TOMOKO SASO $^{4}$, TOMOKI OHTA $^{5}$, \\ KAZUO MIYASHITA ${ }^{1}$ and MASASHI HOSOKAWA ${ }^{1}$
}

\begin{abstract}
${ }^{1}$ Faculty of Fisheries Sciences, Hokkaido University, Hakodate, Hokkaido 041-8611; ${ }^{2}$ Department of Life and Food Sciences, Obihiro University of Agriculture and Veterinary Medicine, Obihiro, Hokkaido 080-8555; ${ }^{3}$ National Food Research Institute, National Agriculture and Food Research Organization, Tsukuba, Ibaraki 305-8642; ${ }^{4}$ Cosmo Co., Ltd., Tokyo 103-0001;

${ }^{5}$ Hokkaido Research Organization, Food Processing Researching Center, Ebetsu, Hokkaido 069-0836, Japan
\end{abstract}

Received July 13, 2020; Accepted October 19, 2020

DOI: 10.3892/ijfn.2020.10

\begin{abstract}
Black chokeberry (Aronia melanocarpa) yields aronia berries, which are rich in anthocyanins. Although aronia juice and its extract have various potential health benefits, information regarding the activity of aronia juice residue is currently limited. The present study examined the effects of aronia juice residue and its ethanol extract on glucose and lipid metabolism in high-fat diet-fed mice. Following a 26-day trial, serum triglycerides, free fatty acids and low-density lipoprotein cholesterol levels were significantly lower in mice fed aronia juice residue or its ethanol extract than in the high-fat-fed control mice. Furthermore, mice fed aronia compounds demonstrated a suppressed hepatic mRNA expression of stearoyl-CoA desaturase 1 , which promotes fatty acid synthesis, whereas the expression levels of cholesterol 7 alpha-hydroxylase, a rate-limiting enzyme of cholesterol catabolism, were increased. Moreover, the ethanol extract inhibited lipase activity in vitro. On the whole, these results indicate that aronia juice residue and its ethanol extract are potentially useful in suppressing lipid metabolic dysfunction in high-fat diet-fed mice.
\end{abstract}

\section{Introduction}

Black chokeberry (Aronia melanocarpa) is a shrub of the Rosaceae family (1). It originates from North America (1) and bears deep-purple fruits approximately 5-10 $\mathrm{mm}$ in diameter. It has been cultivated in Russia, other European countries and

Correspondence to: Dr Nana Mikami, Department of Life and Food Sciences, Obihiro University of Agriculture and Veterinary Medicine, West 2-11, Inada-cho, Obihiro, Hokkaido 080-8555, Japan E-mail: nanam@obihiro.ac.jp

Key words: black chokeberry, Aronia melanocarpa, juice residue, cyanidin-3-galactoside, high-fat diet, lipid metabolism, dyslipidemia more recently, in Japan. The fruits of the black chokeberry (hereafter designated as aronia berries) are excellent sources of anthocyanins and reportedly have the highest anthocyanin content among a variety of commonly consumed foods (2). They are particularly rich in the anthocyanin, cyanidin3 -galactoside (3), and contain various functional components, including carotenoids (i.e., $\beta$-cryptoxanthin, $\beta$-carotene and lutein) and omega-3 fatty acids (i.e., $\alpha$-linolenic acid).

Anthocyanins are associated with a bitter and astringent taste; therefore, aronia berries are generally processed into food products, including juice, jam and wine. Previous reports have demonstrated that aronia juice and its extract have health-promoting properties, such as antioxidant (4-7), anti-hypertensive $(8,9)$ and anti-coagulant $(10)$ properties; they also have the ability to improve glucose levels and lipid metabolism $(11,12)$ and exert hepatoprotective effects $(13)$. During the processing of aronia juice, the residual fruit and peel (hereafter designated as juice residue) remain underutilized, even though they are rich in anthocyanins (14), fiber and other functional components. Therefore, research into the utilization of these by-products is warranted. The authors have previously reported that ethanol extract from aronia juice residue activates lipolysis in adipocytes (15).

Factors, such as a high-fat diet and a sedentary lifestyle induce energy imbalance and fat accumulation in the body, giving rise to obesity. Obesity is a central factor in the development of insulin resistance. Insulin resistance impairs glucose tolerance, leading to the elevation of the plasma concentrations of triglycerides and free fatty acids via the acceleration of the lipogenic pathway (16). Consequently, obesity is closely related to a number of chronic diseases, including diabetes, dyslipidemia, non-alcoholic fatty liver disease and cardiovascular disorders, among others (17). Regular exercise and the control of energy intake are the main recommendations for the prevention and treatment of obesity. However, diet supplementation with functional foods may further promote healthy metabolic function in a convenient and accessible manner.

The present study aimed to investigate whether aronia juice residue and its ethanol extract can improve metabolic 
dysfunction. Thus, the effectiveness of aronia, including its various functional components, on glucose and lipid metabolism in high-fat diet-fed mice were investigated.

\section{Materials and methods}

Materials. Aronia juice residue and ethanol extract (Fig. 1) were obtained from the Hokkaido Research Organization, Food Processing Research Center (Hokkaido, Japan). The nutritional composition of $100 \mathrm{~g}$ of aronia juice residue included: Water, $9.8 \mathrm{~g}$; ash, $1.8 \mathrm{~g}$; protein, $7.4 \mathrm{~g}$; lipid, $7.8 \mathrm{~g}$; and carbohydrate, $73.2 \mathrm{~g}$. Sucrose and L-cystine were purchased from Kanto Chemical Co., Ltd. Choline bitartrate and tert-butylhydroquinone were obtained from Sigma-Aldrich; Merck KGaA. Soybean oil and lard were purchased from Wako Pure Chemical Industries, Ltd. and Hayashi Chemical Industry Co., Ltd., respectively. Other ingredients used for the mouse diets were obtained from CLEA Japan Inc. For the lipase inhibition assay, triolein, pancreatin from porcine pancreas, and Trizma base were purchased from Sigma-Aldrich Japan K.K., whereas sodium taurocholate was obtained from Wako Pure Chemical Industries, Ltd.

Preparation of ethanol extract from aronia juice residue. Freeze-dried aronia juice residue was homogenized using a Polytron homogenizer (Central Scientific Commerce, Inc.) with 20 volumes of $99 \%$ ethanol and extracted overnight. The extract was separated from the residue via filtration utilizing a filter paper (no. 4A) under reduced pressure. This extraction procedure was repeated twice, and the extract was mixed. Excess ethanol in the extract was removed via evaporation under reduced pressure. The yield of the ethanol extraction was $35.7 \%$. The remaining of the extract was used in the animal diets.

Determination of total polyphenol and anthocyanin contents in aronia materials. Total polyphenol and anthocyanin contents in aronia fruit, juice and juice residue were measured using the Folin-Denis method as previously described (18). The anthocyanin content in the aronia ethanol extract was analyzed by high-performance liquid chromatography (HPLC) using the method described in the study by Cassinese et al (19). The SHIMADZU CLASS-VP HPLC system and photodiode-array detector (SPD-M1010Avp) equipped with an Inertsil ODS-3 column (250x4.6 mm i.d., $5 \mu \mathrm{m}$, GL Sciences Inc.) were used. HPLC mobile phases were solvent A (10\% formic acid) and solvent B (10\% formic acid-50\% acetonitrile). Elution was performed as follows: 0-30 $\mathrm{min}, 0-80 \% \mathrm{~B} ; 30-40 \mathrm{~min} 80 \% \mathrm{~B}$. The flow rate was maintained at $1.0 \mathrm{ml} / \mathrm{min}$ at $40^{\circ} \mathrm{C}$. The injection volume of the sample was $20 \mu 1$ and anthocyanin was detected at $520 \mathrm{~nm}$. The total polyphenol and anthocyanin contents were expressed as $\mathrm{mg}$ of chlorogenic acid and cyanidin-3-glucoside equivalent, respectively, in each material. Four anthocyanin content included in the aronia ethanol extract was mg of each anthocyanin using calibration curve by each anthocyanin as external standard method (the contents of cyanidin-3-arabinoside and cyanidin-3-xyloside were quantified by the curve of cyaniding-3-galactoside).

Animals and diets. Male C57BL/6J mice (4 weeks old, 28 mice, $15.5 \mathrm{~g}$ average body weight) were purchased from Charles River
Laboratories Japan, Inc. The mice were housed individually in plastic cages $(13.6 \times 20.8 \times 11.5 \mathrm{~cm})$ under controlled temperature $\left(23 \pm 1^{\circ} \mathrm{C}\right)$, humidity $(45-60 \%)$, and a $12 / 12$-h light and dark cycle, with lights on at 8:00 a.m.; the experimental animals were allowed free access to water and the respective diet throughout the experimental period. The basal diet contained $7 \%$ soybean oil based on the American Institute of Nutrition (AIN-93G) composition (20). Following a 1-week acclimation on the basal diet, the mice were assigned into 4 experimental groups ( 7 mice per group) depending on the different diets given (Table I) as follows: i) The high-fat diet group; ii) high-fat diet with $2 \%$ aronia juice residue; iii) high-fat diet with $1 \%$ aronia ethanol extract; and iv) high-fat diet with $2 \%$ aronia ethanol extract group. The mice were fed their respective diets for 26 days, and were then sacrificed under anesthesia (diethyl ether; $1.9 \%$ diethyl ether for inhalant anesthesia in a glass container) following $12 \mathrm{~h}$ of fasting (9:00 a.m., light period). After a terminal blood sample was collected into a blood collection tube (BD Vacutainer, BD Biosciences), the liver, epididymal, mesenteric, perirenal, retroperitoneal and inguinal white adipose tissues (WATs) were immediately excised and weighed. A specimen of liver tissue was conserved in RNAlater ${ }^{\circledast}$ (Sigma-Aldrich; Merck $\mathrm{KGaA}$ ) for use in reverse transcription-quantitative polymerase chain reaction (PCR) analysis. The animal experiments were approved by the Ethical Committee of Experimental Animal Care at Hokkaido University (Permission no. 09-0094).

Serum glucose and lipid levels. To obtain serum, the collected blood samples were incubated at $25^{\circ} \mathrm{C}$ for $30 \mathrm{~min}$ and then centrifuged at $1,300 \mathrm{x}$ g for $10 \mathrm{~min}$ at $25^{\circ} \mathrm{C}$. The levels of serum lipids [triglycerides, total cholesterol, high-density lipoprotein (HDL) cholesterol, low-density lipoprotein (LDL) cholesterol and free fatty acids] were analyzed at the Hakodate City Medical Association inspection center.

Extraction of total lipids from liver tissue. Total lipids were extracted as previously described by Folch et al (21). Briefly, $100 \mathrm{mg}$ of liver tissue were homogenized with 5 volumes of chloroform-methanol $(2 / 1, \mathrm{v} / \mathrm{v})$ for $2 \mathrm{~min}$, and total lipids were extracted. The extracts were filtered and dried with an evaporator and a vacuum pump.

Measurement of triglyceride and total cholesterol levels in liver tissue. Total lipids extracted from the liver were solubilized by the addition of $200 \mu 1$ Triton X-100-methanol (1/1, $\mathrm{v} / \mathrm{v}$ ) and then dried with nitrogen gas and a vacuum pump. The triglyceride and total cholesterol levels were measured using commercial kits, L type TG Wako and Cholesterol C-test Wako (Wako Pure Chemical Industries, Inc.), respectively, following the manufacturer's instructions.

$R T-q P C R$ for the quantification of $m R N A$ expression in liver tissue. Total RNA was extracted from the liver tissue using the RNeasy Mini kit (Qiagen, Inc.) following the manufacturer's instructions. Briefly, $30 \mathrm{mg}$ of liver tissue in RNAlater was homogenized at 4,000 rpm for $30 \mathrm{sec}$ utilizing Micro Smash (Tomy Seiko Co., Ltd.) with 2 zirconia beads in $300 \mu \mathrm{l}$ RLT buffer.

cDNA was synthesized using total RNA by a reverse transcription reaction with the High-Capacity cDNA Archive 
kit (Applied Biosystems; Thermo Fisher Scientific, Inc.). qPCR was performed with an ABI Prism 7500 sequence detection system (Applied Biosystems; Thermo Fisher Scientific, Inc.). The PCR cycling conditions were as follows: $50^{\circ} \mathrm{C}$ for $2 \mathrm{~min}$ and $95^{\circ} \mathrm{C}$ for $10 \mathrm{~min}$, followed by 40 cycles at $95^{\circ} \mathrm{C}$ for $15 \mathrm{sec}$ and $60^{\circ} \mathrm{C}$ for $1 \mathrm{~min}$. Glyceraldehyde-3-phosphate dehydrogenase (GAPDH), sterol regulatory element-binding protein-1c (SREBP-1c), fatty acid synthase (FAS), stearoyl-CoA desaturase-1 (SCD1), peroxisome proliferator activated receptor $\alpha(\operatorname{PPAR} \alpha)$, long-chain fatty acyl-CoA synthase (ACSL), carnitine palmitoyltransferase 1a (CPT1a), carnitine palmitoyltransferase 2 (CPT2), uncoupling protein 2 (UCP2), hydroxymethylglutaryl-CoA (HMG-CoA) reductase, sterol 14 $\alpha$-demethylase (CYP51), and cholesterol 7 alpha-hydroxylase (CYP7A1) mRNA expression levels were measured using Taq Man Gene Expression Assays (Applied Biosystems; Thermo Fisher Scientific, Inc.). The following PCR primers were purchased from Applied Biosystems; Thermo Fisher Scientific, Inc. for use in these assays: Mm99999915_g1 (Gapdh), Mm00550338_m1 (Srebp-1c), Mm00662319_m1 (Fas), Mm00772290_m1 (Scd1), Mm00440939_m1 (Ppara), Mm00495907_g1 (Ucp2), Mm00550438_m1 (Cpt1a), Mm00487202_m1 (Cpt2), Mm00484217_m1 (Acs11), Mm01282499_m1 (Hmg-coa reductase), Mm00490968_m1 (Cyp51) and Mm00484152_m1 (Cyp7a1).

Lipase inhibition assay. Lipase activity was determined by the amount of free fatty acids released from triolein. The inhibition of lipase activity was calculated based on the reduction in activity following the addition of aronia ethanol extract. The substrate ( $200 \mu \mathrm{l}$ of $10 \mathrm{mM}$ triolein dissolved in $n$-hexane) was placed into a glass vial, and the solvent was removed using nitrogen gas. The substrate emulsion was prepared by sonication of triolein with $1 \mathrm{ml}$ of $20 \mathrm{mM}$ sodium taurocholate and $0,2.5,5.0,7.5$, or $10.0 \mathrm{mg} / \mathrm{ml}$ of aronia ethanol extract in $100 \mathrm{mM}$ Tris- $\mathrm{HCl}$ buffer ( $\mathrm{pH} \mathrm{8.0)}$ for $5 \mathrm{~min}$. The mixture was incubated with $1 \mathrm{ml}$ lipase solution $[10 \mathrm{mg} / \mathrm{ml}$ pancreatin from porcine pancreas (Wako Pure Chemical Industries) in $100 \mathrm{mM}$ Tris- $\mathrm{HCl}$ buffer $\left(\mathrm{pH} \mathrm{8.0)}\right.$ ] for $6 \mathrm{~h}$ at $37^{\circ} \mathrm{C}$ with a magnetic stirrer. The enzyme reaction was terminated by the addition of 2 volumes $n$-hexane and centrifugation at $550 \mathrm{x} \mathrm{g}$ for $5 \mathrm{~min}$ at $25^{\circ} \mathrm{C}$. The amount of free fatty acids was quantified by colorimetry, with the absorbance of the $n$-hexane fraction at $550 \mathrm{~nm}$ using the NEFA C-test Wako kit (Wako Pure Chemical Industries).

Statistical analysis. Data are expressed as the means \pm standard deviation (SD) or as box-and-whisker plots ( $\mathrm{n}=7$ mice per group for in vivo experiments and $\mathrm{n}=3$ per group for in vitro experiments). Statistical significance was determined by one-way analysis of variance (ANOVA). When statistically significant differences $(\mathrm{P}<0.05)$ were observed, Dunnett's test was used for multiple comparisons. $\mathrm{P}<0.05$ or $\mathrm{P}<0.01$ were considered to indicate statistically significant differences. Data were analyzed utilizing Excel Tokei software 6.0 (Esumi Co., Ltd.).

\section{Results}

Aronia berries are known as excellent sources of polyphenols and particularly, anthocyanins. First, the present study

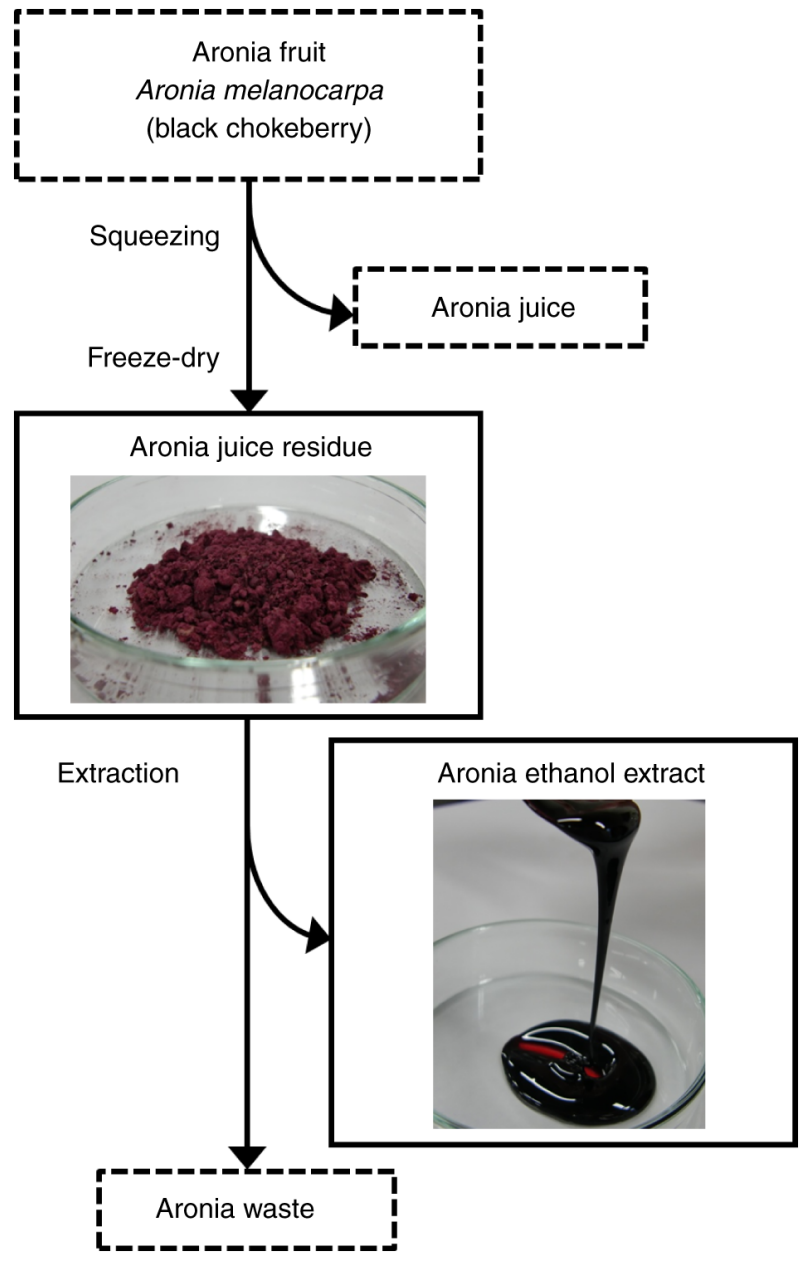

Figure 1. Flow chart of aronia processing.

determined the total polyphenol and anthocyanin contents in the aronia materials used. The total polyphenol contents of aronia fruit, juice and juice residue were 491.4 \pm 52.2 , $57.5 \pm 0.78$ and $433.9 \pm 51.4 \mathrm{mg} / 100 \mathrm{~g}$ dry matter, respectively (Table II). The distribution of the anthocyanin content in each material demonstrated an almost equal ratio to that of the total polyphenol content. These results indicate that the majority of polyphenols and anthocyanins of the aronia fruit remain in its juice residue instead of in the juice when the fruit is squeezed. Anthocyanins in aronia fruit, juice and juice residue accounted for almost all of the total polyphenol content. Furthermore, the anthocyanin content in the aronia ethanol extract was $1446.7 \pm 63.4 \mathrm{mg} / 100 \mathrm{~g}$ dry matter and was 3.4-fold times higher than that in the juice residue. The aronia ethanol extract included several glycosides of cyanidin, predominantly cyanidin-3-galactoside and cyanidin3 -arabinoside $(886.7 \pm 4.7 \mathrm{mg}$ and $456.7 \pm 41.1 \mathrm{mg} / 100 \mathrm{~g}$ dry matter, respectively) (Table II).

To utilize these potential aronia by-products, the effects of aronia juice residue and its ethanol extract on glucose and lipid metabolism were evaluated through the administration of high-fat diets, including aronia by-products, to C57BL/6J mice.

Following the experimental feeding period, the final body weights of the mice did not differ significantly among the mice from the 4 experimental groups (Table III), although the food intake was higher in the aronia juice residue group than in the 
Table I. Composition of the 4 experimental diets used in the present study.

\begin{tabular}{|c|c|c|c|c|}
\hline \multirow[b]{3}{*}{ Ingredients $(\mathrm{g} / \mathrm{kg})$} & \multirow[b]{3}{*}{ High-fat diet } & \multicolumn{3}{|c|}{ Aronia } \\
\hline & & \multirow{2}{*}{$\frac{\text { Juice residue }}{2 \%}$} & \multicolumn{2}{|c|}{ Ethanol extract } \\
\hline & & & $1 \%$ & $2 \%$ \\
\hline Cornstarch & 181.28 & 166.27 & 181.28 & 181.28 \\
\hline Casein & 258.00 & 258.00 & 258.00 & 258.00 \\
\hline Dextrized cornstarch & 60.20 & 55.21 & 60.20 & 60.20 \\
\hline Sucrose & 100.00 & 100.00 & 100.00 & 100.00 \\
\hline Soybean oil & 70.00 & 70.00 & 60.00 & 50.00 \\
\hline Lard & 230.00 & 230.00 & 230.00 & 230.00 \\
\hline Cellulose & 50.00 & 50.00 & 50.00 & 50.00 \\
\hline Mineral mix (AIN-93G-MX) & 35.00 & 35.00 & 35.00 & 35.00 \\
\hline Vitamin mix (AIN-93G-VX) & 10.00 & 10.00 & 10.00 & 10.00 \\
\hline L-cystine & 3.00 & 3.00 & 3.00 & 3.00 \\
\hline Choline bitartrate & 2.50 & 2.50 & 2.50 & 2.50 \\
\hline tert-Butylhydroquinone & 0.02 & 0.02 & 0.02 & 0.02 \\
\hline Aronia juice residue & - & 20.00 & - & - \\
\hline Aronia ethanol extract & - & - & 10.00 & 20.00 \\
\hline
\end{tabular}

Table II. Total polyphenol and anthocyanin contents in aronia materials.

\begin{tabular}{llccccc}
\hline Polyphenols/anthocyanins & & \multicolumn{4}{c}{ Aronia } \\
\cline { 1 - 1 } \cline { 6 - 7 } Polyphenols (mg/100 g dry matter) & & Fruits & & Juice & Juice residue & Ethanol extract \\
\hline Total polyphenol content (chlorogenic acid equivalent) & & $491.4 \pm 52.2$ & $57.5 \pm 0.78$ & $433.9 \pm 51.4$ & \\
Anthocyanin content (cyanidin 3-glucoside equivalent) & & $486.2 \pm 45.4$ & $57.1 \pm 0.76$ & $429.1 \pm 4.6$ & $1446.7 \pm 63.4$ \\
\hline
\end{tabular}

Anthocyanins (mg/100 g dry matter)

Ethanol extract

Cyanidin-3-galactoside

$886.7 \pm 4.7$

Cyanidin-3-arabinoside

$456.7 \pm 41.1$

Cyanidin-3-xyloside

$60.0 \pm 8.2$

Cyanidin 3-glucoside

$43.3 \pm 9.4$

Values are expressed as the means $\pm \mathrm{SD}, \mathrm{n}=3$.

high-fat diet group. Furthermore, the administration of aronia juice residue and ethanol extract did not significantly change the weight of the liver and white adipose tissues (Table III). The blood glucose levels were decreased in mice fed the aronia juice residue $(107.0 \pm 16.8 \mathrm{mg} / \mathrm{dl})$ or ethanol extracts $(102.3 \pm 17.7$ and $101.6 \pm 32.4 \mathrm{mg} / \mathrm{dl}$ for 1 and $2 \%$ extract, respectively) compared to those in mice fed the high-fat diet (130.7 $\pm 44.8 \mathrm{mg} / \mathrm{dl})$, although differences among the groups did not reach statistical significance (Table III).

The serum triglyceride levels were significantly lower in mice fed the $2 \%$ aronia juice residue $(26.1 \pm 3.7 \mathrm{mg} / \mathrm{dl})$, and 1 and $2 \%$ aronia ethanol extract diets $(39.4 \pm 12.5$ and $33.0 \pm 12.4 \mathrm{mg} / \mathrm{dl}$, respectively) than those in the high-fat diet-fed mice $(70.1 \pm 23.3 \mathrm{mg} / \mathrm{dl})$ (Fig. 2A). Furthermore, the serum free fatty acid levels were decreased in the $2 \%$ juice residue and $2 \%$ aronia ethanol extract groups compared to the high-fat diet group (Fig. 2B). Although the serum free fatty acid levels in the $1 \%$ ethanol extract group were reduced compared to those in the high-fat diet group, the difference was not significant.

The total cholesterol levels in serum did not differ significantly among the 4 groups (Fig. 3A). However, the HDL cholesterol levels were significantly lower in mice the fed $1 \%$ aronia ethanol extract diet than in the mice fed the high-fat diet (Fig. 3B). The levels of LDL cholesterol were significantly lower in the mice fed the aronia juice residue, and the 1 and $2 \%$ aronia ethanol extract diets than in the mice fed the high-fat $\operatorname{diet}$ (Fig. 3C).

Due to the observed reductions in triglycerides, free fatty acids and the LDL cholesterol content, the levels of total hepatic 
Table III. Effects of aronia juice residue and ethanol extract on body parameters, food intake and blood glucose levels in C57/BL6J mice fed the fed high-fat diet.

\begin{tabular}{|c|c|c|c|c|}
\hline \multirow[b]{3}{*}{ Parameter } & \multirow[b]{3}{*}{ High-fat diet } & \multicolumn{3}{|c|}{ Aronia } \\
\hline & & \multirow{2}{*}{$\frac{\text { Juice residue }}{2 \%}$} & \multicolumn{2}{|c|}{ Ethanol extract } \\
\hline & & & $1 \%$ & $2 \%$ \\
\hline Final body weight (g) & $27.2 \pm 1.0$ & $28.0 \pm 2.2$ & $27.9 \pm 1.8$ & $27.0 \pm 1.5$ \\
\hline Food intake (g/26 days) & $77.4 \pm 9.3$ & $90.9 \pm 6.5^{\mathrm{a}}$ & $81.5 \pm 4.1$ & $86.5 \pm 11.6$ \\
\hline Liver (g/100 g body weight) & $3.9 \pm 0.4$ & $3.7 \pm 0.2$ & $3.6 \pm 0.1$ & $3.9 \pm 0.1$ \\
\hline White adipose tissues ( $\mathrm{g} / 100 \mathrm{~g}$ body weight) & $5.7 \pm 0.7$ & $6.2 \pm 1.2$ & $5.9 \pm 0.8$ & $5.9 \pm 0.7$ \\
\hline Blood glucose level (mg/dl) & $130.7 \pm 44.8$ & $107.0 \pm 16.8$ & $102.3 \pm 17.7$ & $101.6 \pm 32.4$ \\
\hline
\end{tabular}

Values are expressed as the means $\pm \mathrm{SD}, \mathrm{n}=7$. White adipose tissue; total weight of mesenteric, epididymal, perirenal and retroperitoneal white adipose tissues. ${ }^{a} \mathrm{P}<0.05$, significant difference compared with the high-fat diet-fed group.
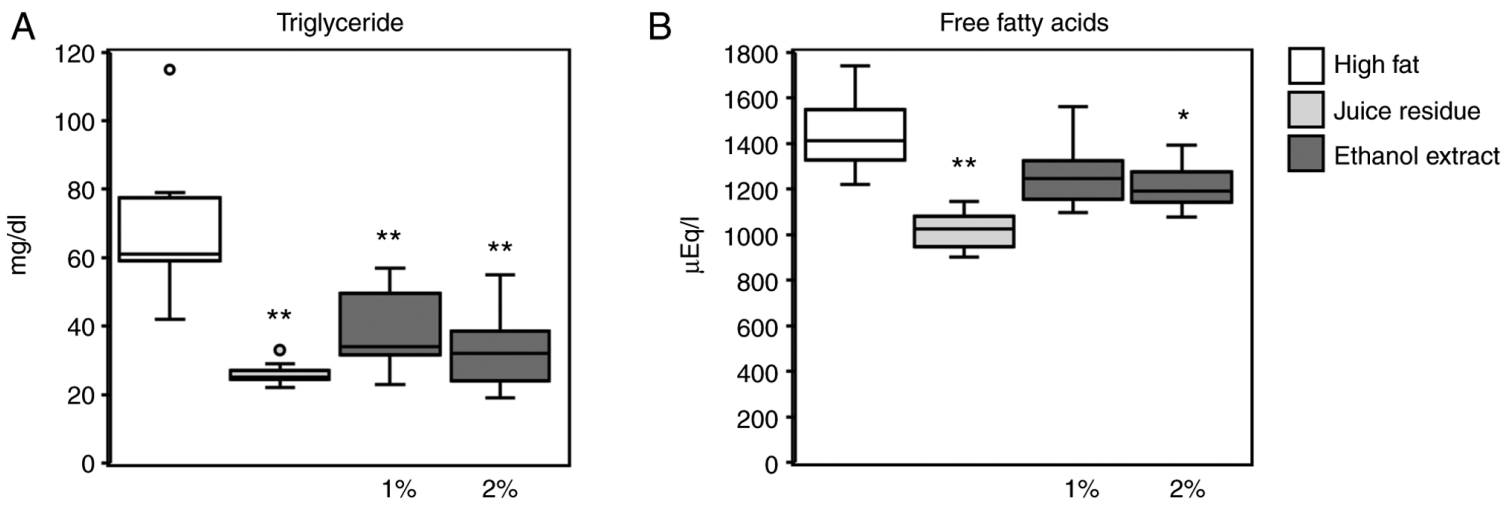

Figure 2. Effect of aronia juice residue and its ethanol extract on serum triglyceride and free fatty acid levels in C57BL/6J mice fed a high-fat diet. (A) Serum triglyceride levels were significantly decreased in mice fed aronia juice residue and its ethanol extract compared to the high-fat diet-fed control mice. (B) Serum free fatty acid levels were significantly decreased in mice fed aronia juice residue and $2 \%$ ethanol extract compared to the high-fat diet-fed control mice. Data are presented in the form of box and whisker plots, wherein the central box represents levels in the lower and upper interquartile range, and the middle line marks the median $(n=7)$. The maximum and minimum levels for each group are represented by each end of the whiskers, and outliers are delineated by circles. Significant differences were compared among the 4 groups and the control. ${ }^{*} \mathrm{P}<0.05$ and ${ }^{* *} \mathrm{P}<0.01$, significant difference compared with the high-fat diet-fed group.
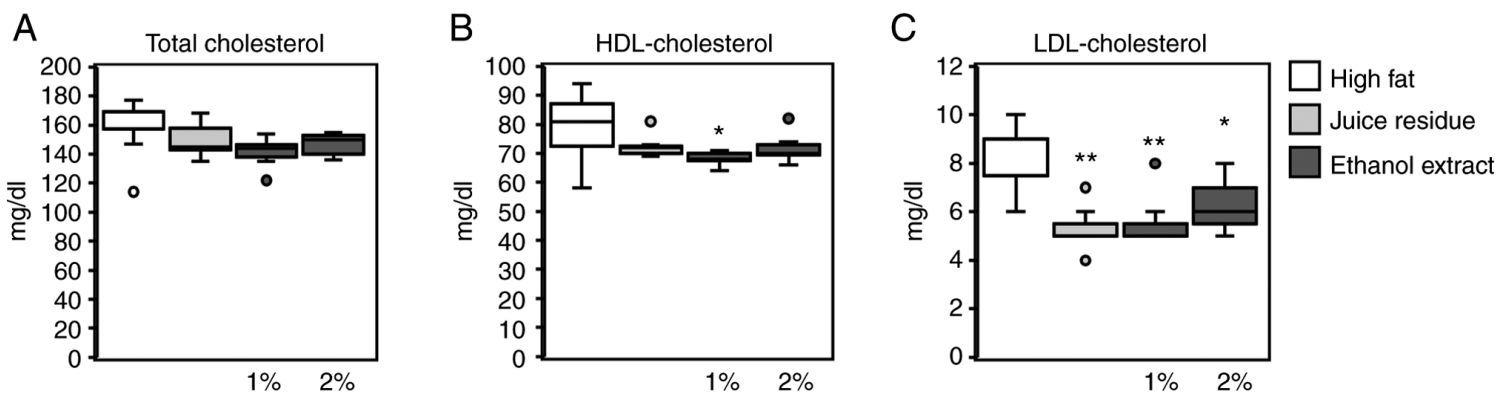

Figure 3. Effect of aronia juice residue and its ethanol extract on serum cholesterol (total cholesterol, HDL cholesterol and LDL cholesterol) levels in C57BL/6J mice fed a high-fat diet. (A) Total cholesterol levels in serum did not differ significantly among the 4 groups. (B) HDL cholesterol levels were significantly lower in the $1 \%$ aronia ethanol extract group than in the high-fat diet-fed group. (C) LDL cholesterol were significantly lower in the aronia juice residue and 1 and $2 \%$ aronia ethanol extract groups than in the high-fat diet-fed group. Data are presented in the form of box and whisker plots, wherein the central box represents levels in the lower and upper interquartile range, and the middle line marks the median $(n=7)$. The maximum and minimum levels for each group are represented by each end of the whiskers, and outliers are delineated by circles. Significant differences were compared among the 4 groups and the control. ${ }^{*} \mathrm{P}<0.05$ and $^{* *} \mathrm{P}<0.01$, significant difference compared with the high-fat diet-fed group. HDL, high-density lipoprotein; LDL, low-density lipoprotein.

lipids, triglycerides and cholesterol were measured (Table IV). The total liver lipid and triglyceride content did not differ significantly among the 4 experimental groups. Significant increases $(\mathrm{P}<0.05)$ in total cholesterol levels were observed 
Table IV. Levels of total lipids, triglycerides and total cholesterols in the livers of C57BL/6J mice fed the high-fat diet with aronia juice residue and ethanol extract.

\begin{tabular}{|c|c|c|c|c|}
\hline \multirow[b]{3}{*}{ Parameter } & \multirow[b]{3}{*}{ High-fat } & \multicolumn{3}{|c|}{ Aronia } \\
\hline & & \multirow{2}{*}{$\frac{\text { Juice residue }}{2 \%}$} & \multicolumn{2}{|c|}{ Ethanol extract } \\
\hline & & & $1 \%$ & $2 \%$ \\
\hline Total lipids ( $\mathrm{mg} / \mathrm{g}$ liver) & $73.2 \pm 14.7$ & $115.3 \pm 43.4$ & $72.2 \pm 34.7$ & $104.1 \pm 36.3$ \\
\hline Triglycerides (mg/g liver) & $22.5 \pm 10.4$ & $20.0 \pm 7.1$ & $20.1 \pm 9.7$ & $19.1 \pm 6.9$ \\
\hline Total cholesterols (mg/g liver) & $4.05 \pm 1.00$ & $6.15 \pm 1.47^{\mathrm{a}}$ & $6.26 \pm 2.03^{\mathrm{a}}$ & $5.63 \pm 1.49$ \\
\hline
\end{tabular}

Values are expressed as the means $\pm \mathrm{SD}, \mathrm{n}=7 .{ }^{\mathrm{a}} \mathrm{P}<0.05$, significant difference compared with the high-fat diet-fed group.
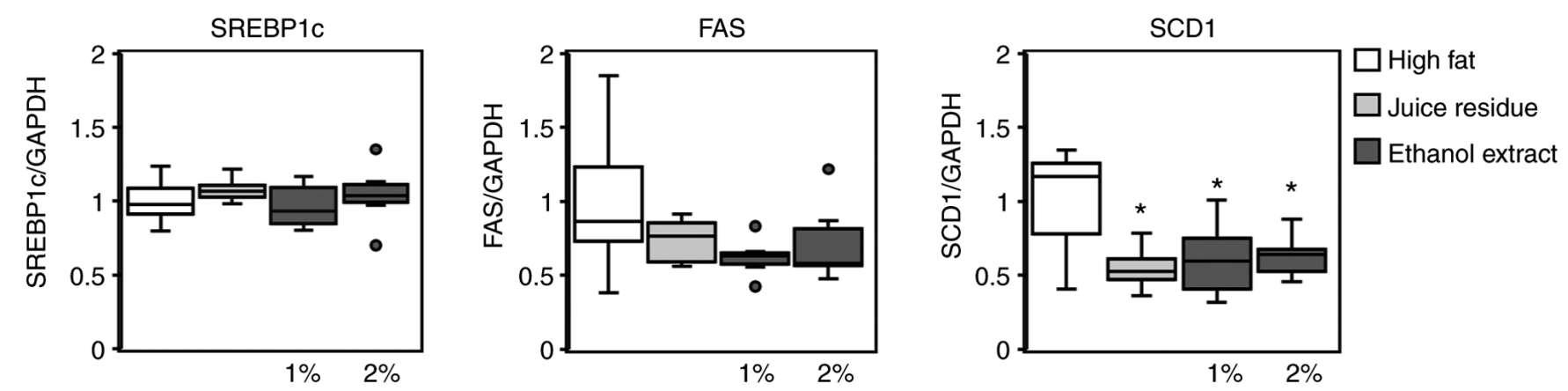

Figure 4. Effect of aronia juice residue and its ethanol extract on hepatic mRNA expression levels of genes involved in fatty acid synthesis in C57BL/6J mice fed a high-fat diet. SREBP-1c and FAS expression levels did not differ significantly among the 4 groups. SCD1 expression levels decreased significantly in the aronia juice residue and ethanol extract groups compared to high-fat diet-fed group. Data are presented in the form of box and whisker plots, wherein the central box represents levels in the lower and upper interquartile range, and the middle line marks the median ( $\mathrm{n}=6-7)$. The maximum and minimum levels for each group are represented by each end of the whiskers, and outliers are delineated by circles. Significant differences were compared among the 4 groups and the control. " $\mathrm{P}<0.05$, significant difference compared with the high-fat diet-fed group. SREBP-1c, sterol regulatory element-binding protein-1c; FAS, fatty acid synthase; SCD1, stearoyl-CoA desaturase-1.

in the livers of the mice fed the aronia juice residue and $1 \%$ ethanol extract compared to the mice fed the high-fat diet (Table IV). The mice fed the $2 \%$ ethanol extract also exhibited an increase in total cholesterol levels compared to the mice fed the high-fat diet, although the difference was not significant.

To further investigate the effects of aronia juice residue and its ethanol extract on lipid metabolism, the hepatic expression levels of genes involved in lipid synthesis and degradation were measured. As shown in Fig. 4, the hepatic mRNA levels of genes implicated in lipogenesis (i.e., SREBP-1c, FAS and SCD1) were determined. SREBP-1c expression was not affected by the administration of aronia juice residue or ethanol extract (Fig. 4). By contrast, the FAS and SCD1 mRNA levels were decreased in mice fed the aronia juice residue or its ethanol extract compared to the mice fed the high-fat diet, although the differences in FAS expression were not significant (Fig. 4). In addition, the hepatic expression levels of genes associated with lipolysis were measured (Fig. 5). The PPAR $\alpha$ mRNA levels did not differ significantly among the 4 experimental groups. However, the expression of ACSL, CPT1a and CPT2 was significantly upregulated in the mice fed the $1 \%$ aronia ethanol extract compared to the mice fed the high-fat diet (Fig. 5). Of note, CPT1a was the only gene whose expression was significantly increased in the mice fed the $2 \%$ ethanol extract. Moreover, UCP2 expression was significantly upregulated in the mice fed the aronia juice residue compared to the mice fed the high-fat diet (Fig. 5).

To determine the cause of the observed decrease in serum LDL cholesterol levels in mice fed the aronia juice residue or its ethanol extract, the hepatic mRNA levels of genes involved in cholesterol metabolism were measured. As shown in Fig. 6, the expression levels of HMG-CoA reductase and CY P51, which are involved in cholesterol synthesis, were not significantly affected by the inclusion of aronia in the diet (Fig. 6). On the contrary, the mRNA levels of CYP7A1, which is a rate-limiting enzyme in the hepatic catabolism of cholesterol into bile acid, were markedly and significantly increased with the inclusion of aronia juice residue (3.7-fold) and $1 \%$ or $2 \%$ ethanol extract (2.5- or 3.3-fold, respectively) in the diet compared to the high-fat diet.

Subsequently, to investigate whether aronia ethanol extract affects triglyceride absorption, the inhibition of lipase activity in vitro was evaluated (Fig. 7). Ethanol extract of aronia juice residue lowered the release of free fatty acids from triolein in a dose-dependent manner with significant decreases observed at the $5,7.5$ and $10 \mathrm{mg} / \mathrm{ml}$ concentrations. The $\mathrm{IC}_{50}$ value for pancreatic lipase was $4.6 \mathrm{mg} / \mathrm{ml}$ ethanol extract. 

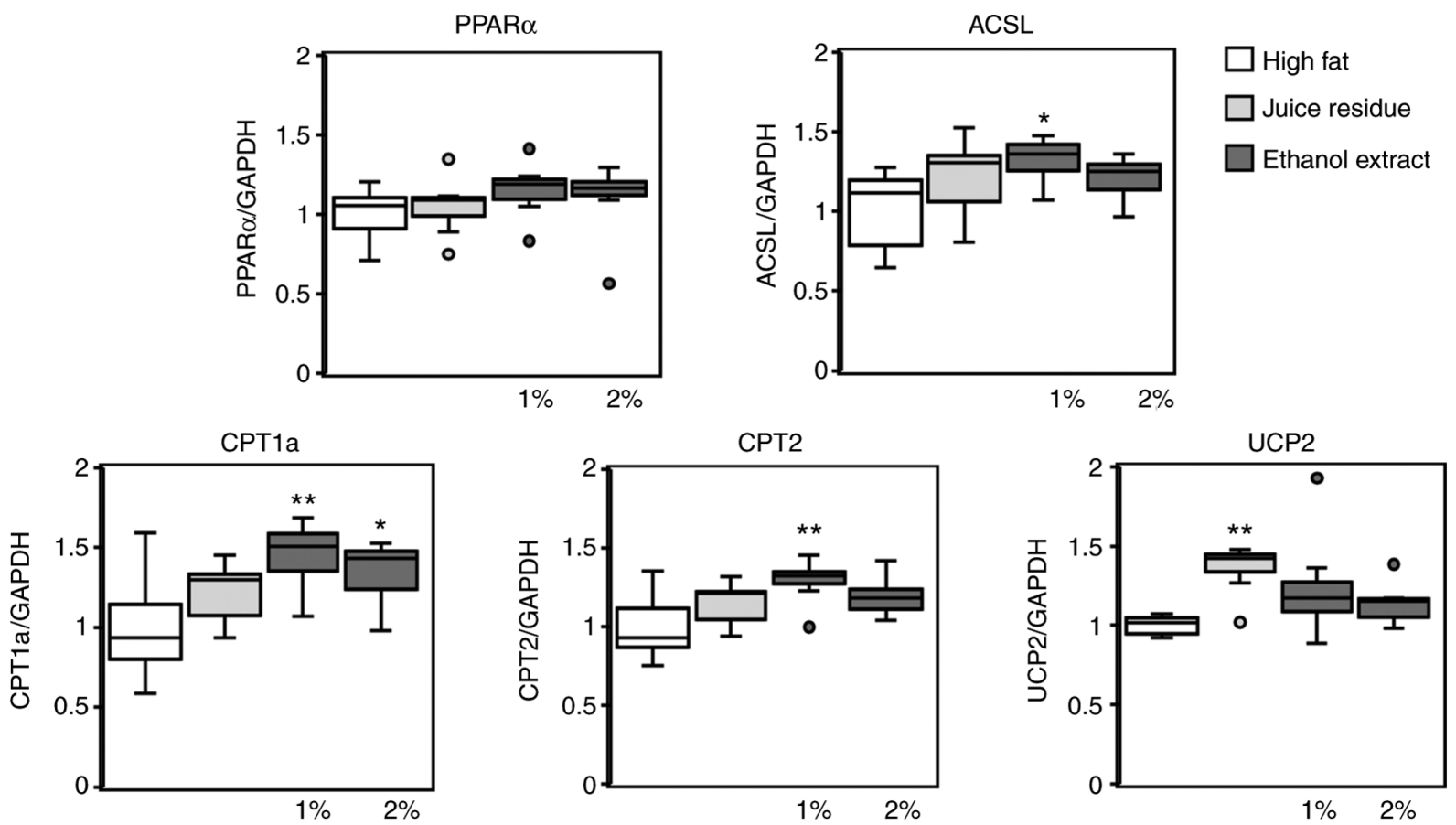

Figure 5. Effect of aronia juice residue and its ethanol extract on hepatic mRNA expression levels of genes involved in fatty acid oxidation in C57BL/6J mice fed a high-fat diet. PPAR $\alpha$ expression did not differ significantly among the 4 groups. ACSL, CPT1a and CPT2 expression levels were significantly upregulated in the $1 \%$ aronia ethanol extract group compared to high-fat group. CPT1a was the only gene whose expression was significantly increased in $2 \%$ ethanol extract group. UCP2 expression was significantly upregulated in aronia juice residue group compared to the high-fat diet-fed group. Data are presented in the form of box and whisker plots, wherein the central box represents levels in the lower and upper interquartile range, and the middle line marks the median $(\mathrm{n}=7)$. The maximum and minimum levels for each group are represented by each end of the whiskers, and outliers are delineated by circles. Significant differences were compared among the 4 groups and the control. ${ }^{*} \mathrm{P}<0.05$ and ${ }^{* *} \mathrm{P}<0.01$, significant difference compared with the high-fat diet-fed group. PPAR $\alpha$, peroxisome proliferator activated receptor $\alpha$; ACSL, long-chain fatty acyl-CoA synthase; CPT1a, carnitine palmitoyltransferase 1a; CPT2, carnitine palmitoyltransferase 2 .
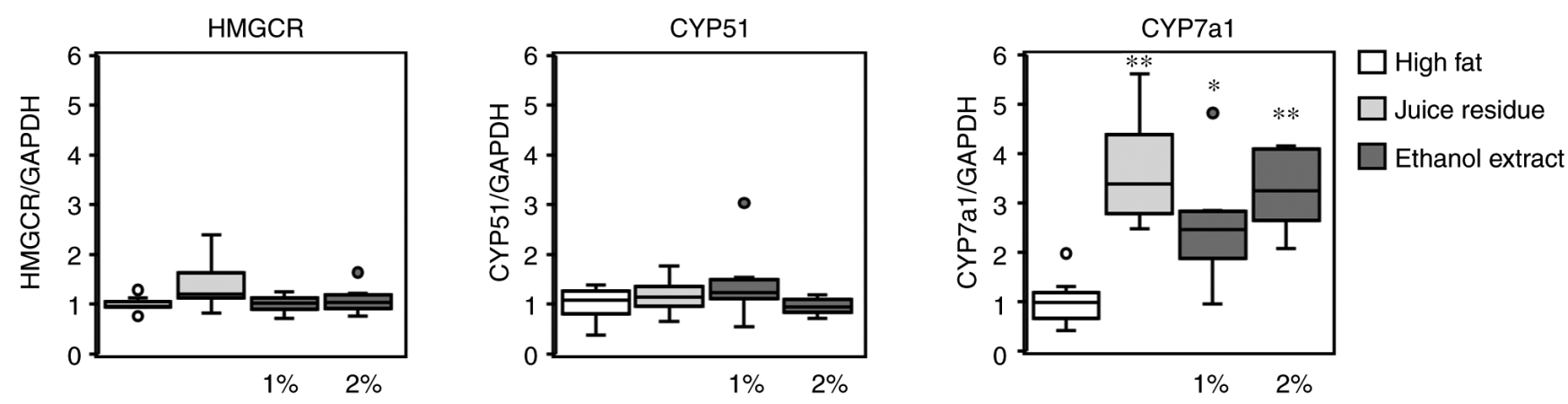

Figure 6. Effect of aronia juice residue and its ethanol extract on hepatic mRNA expression levels of genes involved in cholesterol metabolism in C57BL/6J mice fed a high-fat diet. HMG-CoA reductase and CYP51 expression levels did not differ among the 4 groups. CYP7A1 expression levels significantly increased in aronia juice residue and $1 \%$ or $2 \%$ ethanol extract groups compared to high-fat group. Data are presented in the form of box and whisker plots, wherein the central box represents levels in the lower and upper interquartile range, and the middle line marks the median $(\mathrm{n}=7)$. The maximum and minimum levels for each group are represented by each end of the whiskers, and outliers are delineated by circles. Significant difference was compared among the 4 groups and the control. " $\mathrm{P}<0.05$ and ${ }^{* *} \mathrm{P}<0.01$, significant difference compared with the high-fat diet-fed group. HMGCR/HMG-CoA, hydroxymethylglutaryl-CoA reductase; CYP51, sterol 14 alpha-demethylase; CYP7A1, cholesterol 7 alpha-hydroxylase.

\section{Discussion}

Black chokeberry has numerous potential health benefits due to its high levels of anthocyanins and other functional compounds. The processed juice residue is also rich in these compounds, but is currently underutilized due to a lack of research into its health-promoting effects and functionality.

The objective of the present study was to determine the bio-functional effects of aronia juice residue and its ethanol extract on glucose and lipid metabolism in high-fat diet-fed mice. Following a 26-day feeding trial involving mice on a high-fat diet or a high-fat diet supplemented with aronia juice residue $(2 \%)$ or ethanol extract $(1 \%$ or $2 \%)$, no significant differences were detected in body weight, liver weight WAT weight, or blood glucose levels among the 4 groups. However, serum triglyceride, free fatty acid and LDL-cholesterol levels were significantly lower in mice fed aronia juice residue and ethanol extract than in mice fed a high-fat control diet. These 


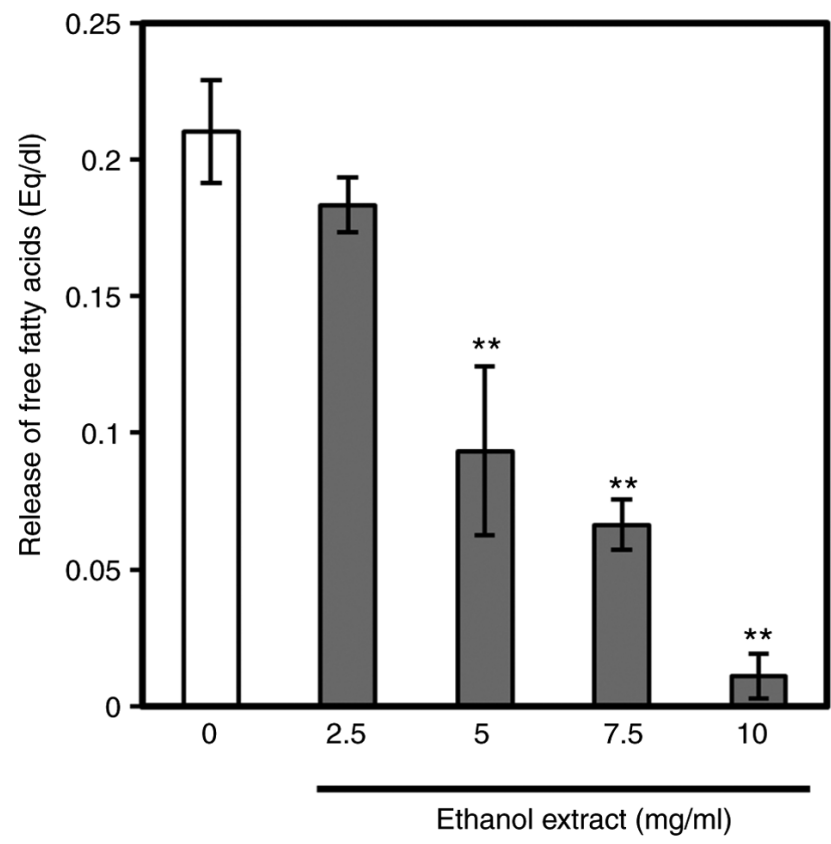

Figure 7. Effect of ethanol extract of aronia juice residue on lipase inhibition, based on the release of free fatty acids. Ethanol extract of aronia juice residue lowered the release of free fatty acids from triolein in a dose-dependent manner with significant reductions at the $5,7.5$ and $10 \mathrm{mg} / \mathrm{ml}$ concentrations. Values are expressed as the means \pm SD $(n=3)$. Significant differences were compared among the 5 concentrations and the control. ${ }^{* *} \mathrm{P}<0.01$, significant difference compared with the control $(0 \mathrm{mg})$.

data suggest that the administration of aronia juice residue or its ethanol extract prevents the development of elevated serum lipid profiles in high-fat diet-fed mice.

Dyslipidemia, such as hyper-LDL cholesterolemia and hypertriglyceridemia, is one of the health issues associated with obesity and leads to arteriosclerosis. Takahashi et al (12) previously reported that a 4 -week feeding period with $1.7 \%$ aronia phytochemicals (i.e., $0.4 \%$ anthocyanins) suppressed visceral fat accumulation in mice fed a high-fat diet. Furthermore, this treatment decreased serum LDL cholesterol and glucose levels (12). In the present study, supplementation with aronia juice residue or its ethanol extract did not affect visceral fat accumulation or blood glucose levels; however, the results of the present are consistent with those of the study by Takahashi et al (12) in terms of the LDL cholesterol reduction as a result of aronia supplementation. Moreover, Valcheva-Kuzmanova et al (22) reported that the administration of aronia fruit juice suppressed the plasma total cholesterol, LDL cholesterol and triglyceride levels. As indicated by serum triglyceride and LDL cholesterol levels, these parameters may be improved by common components of aronia juice and its residue (i.e., anthocyanins and carotenoids). Furthermore, aronia juice residue contains a considerable amount of mainly insoluble dietary fibers (23). A previous study found that the insoluble, fiber-rich fraction of carrot pomace lowered serum triglyceride and total cholesterol levels in hamsters (24). The aronia juice residue used in the present study contained anthocyanin and carbohydrate $(429.1 \mathrm{mg}$ and $73.2 \mathrm{~g}$ in $100 \mathrm{~g}$ dry matter, respectively). The present study did not determine the dietary fiber content in aronia juice residue; however, a previous study demonstrated that dietary fiber accounted for approximately $40 \%$ of carbohydrate in aronia fruit (25). Therefore, the observed improvement in lipid metabolism in the present study may have occurred due to the combined effects of the functional components of aronia juice residue, such as anthocyanin and dietary fibers.

Lipid metabolism is primarily controlled by a combination of hepatic enzymes and transcription factors. SREBP-1c is a master regulator of de novo lipogenesis and regulates hepatic transcription of lipogenic enzymes, such as FAS and SCD1 (26). A high-fat diet induces the expression of these lipogenic genes in the liver (27). In a previous study, Park et al (28) reported that an 8 -week administration of $1 \%$ aronia powder with a high-fat diet decreased the hepatic mRNA expression of SREBP1 and FAS in mice with non-alcoholic fatty liver disease. In the present study, aronia juice residue and its ethanol extract did not affect hepatic SREBP1 expression. However, the SCD1 mRNA levels decreased significantly in mice fed high-fat diets supplemented with aronia compared to the high-fat diet-fed control mice. These results suggest that aronia juice residue and its ethanol extract suppressed de novo lipogenesis through a mechanism independent of SREBP-1c, contrary to the findings of the study by Park et al (28). In terms of hepatic lipolysis-related mRNA expression, PPAR $\alpha$ levels, a master regulator of fatty acid oxidation, were not affected by aronia juice residue or its ethanol extract. However, the mRNA levels of ACSL, CPT1a and CPT2, which are enzymes targeted by PPAR $\alpha$ in the promotion of acyl CoA formation or mitochondrial $\beta$-oxidation (29), were significantly increased in mice fed a high-fat diet supplemented with aronia ethanol extract in the present study. Furthermore, supplementation with aronia juice residue increased the UCP 2 mRNA levels, without affecting the PPAR $\alpha$ levels, even though UCP2 gene expression in hepatocytes is upregulated by $\operatorname{PPAR} \alpha$ activators (30). The present findings indicate that aronia juice residue and its ethanol extract regulate these genes without affecting the PPAR $\alpha$ pathway. These findings also suggest that aronia juice residue and its ethanol extract reduce serum triglyceride and free fatty acid levels through distinct mechanisms by altering the expression of different hepatic genes. In the present study, a dose-dependent decrease in serum triglyceride and free fatty acid levels was observed with the administration of 1 and $2 \%$ ethanol extracts with the diet. However, the effect on hepatic gene expression, and particularly, on lipolysis-related enzymes, was not dose-dependent. Further studies are required to elucidate the mechanism through which hepatic lipogenic and lipolytic gene expression is involved in the observed ethanol extract-induced decrease in serum triglyceride and free fatty acid levels.

Another mechanism that may explain the changes detected in the serum lipid profile may be the inhibition of dietary lipid absorption. Pancreatic lipase hydrolyzes triglycerides into fatty acids and glycerols and is responsible for the hydrolysis of $50-70 \%$ of total dietary fats (31). Dietary fat is absorbed by the small intestine, only when it has been hydrolyzed by a pancreatic lipase (32). Therefore, the inhibition of lipase activity is a potential mechanism for the regulation of serum triglyceride and free fatty acid levels. In the present study, the ethanol extract of aronia juice residue inhibited lipase activity in a dose-dependent manner. Previous studies have demonstrated that anthocyanin-rich phytochemicals from aronia 
berries and polyphenol-rich extracts from various berries also inhibit lipase activity $(12,33)$. The present data revealed that aronia ethanol extract was rich in anthocyanins $(1.45 \mathrm{~g} / 100 \mathrm{~g}$ dry matter, Table II), including cyanidin-3-galactoside and cyanidin-3-arabinoside. Based on these results, the improved serum lipid profile observed in the present study may have been partially caused by the inhibition of pancreatic lipase due to anthocyanins in the ethanol extract of aronia juice residue.

The results of the present study also demonstrate a decrease in serum LDL cholesterol in high-fat diet-fed mice as a result of supplementation with aronia juice residue or its ethanol extract. Furthermore, the total hepatic cholesterol levels increased in mice fed aronia juice residue or $1 \%$ ethanol extract. Circulating cholesterol levels are regulated by a balance between cholesterol biosynthesis, catabolism and transfer into peripheral tissues. Focusing on cholesterol biosynthesis in the liver, the mRNA levels of HMG-CoA reductase and CYP51, which are involved in cholesterol synthesis, were not affected by supplementation with aronia juice residue and its ethanol extract. However, the expression of CYP7A1, which is a rate-limiting enzyme that converts cholesterol to bile acid, markedly increased with the administration of aronia juice residue or ethanol extract. Previous studies have also revealed the upregulation of hepatic CYP7A1 expression by anthocyanin (34) and procyanidin administration (35). These data suggest that the aronia juice residue and ethanol extract containing anthocyanins that were used in our study may regulate serum LDL-cholesterol through hepatic cholesterol excretion, not cholesterol synthesis. Furthermore, the decrease in plasma LDL cholesterol levels is caused by an increased LDL receptor activity (36). A previous study revealed that red grape juice with anthocyanins upregulated both the activity and expression of the LDL receptor and total cholesterol content in HepG2 cells in the presence of LDL (37). Although the present study did not evaluate the activity of the hepatic LDL receptor, these findings raise the possibility that aronia juice residue and its ethanol extract may decrease serum LDL-cholesterol levels by incorporating cholesterol into the liver via LDL receptor activation.

In conclusion, aronia juice residue and its ethanol extract exhibited anti-hypolipidemic activities in high-fat diet-fed mice by the regulation of hepatic gene expression related to fatty acid synthesis, fatty acid oxidation, cholesterol degradation and lipase inhibition. The results suggest that the underutilized aronia juice residue and its ethanol extract may be potentially used as ingredients in the functional food industry. However, there are some limitations, including the lack of measuring protein expression and enzyme activities in lipid metabolic pathway. Further research is required to determine their effectiveness in more detail and the optimal applications for their utilization in foods.

\section{Acknowledgements}

Not applicable.

\section{Funding}

No funding was received.

\section{Availability of data and materials}

All data used during the present study are available from the corresponding author on reasonable request.

\section{Authors' contributions}

$\mathrm{YH}, \mathrm{TO}$ and $\mathrm{MH}$ performed the experiments in this study. NM, YH, TS, TO, KM and MH contributed to the design and interpretation of the study, as well as in the writing and revision of the manuscript. All authors read and approved the final manuscript.

\section{Ethics approval and consent to participant}

All animal procedures were carried out according to the protocol approved by the Ethical Committee of Experimental Animal Care at Hokkaido University (Permission no. 09-0094) in Japan.

\section{Patient consent for publication}

Not applicable.

\section{Competing interests}

The authors declare that they have no competing interests.

\section{References}

1. Kokotkiewicz A, Jaremicz Z and Luczkiewicz M: Aronia plants: A review of traditional use, biological activities, and perspectives for modern medicine. J Med Food 13: 255-269, 2010.

2. Wu X, Beecher GR, Holden JM, Haytowitz DB, Gebhardt SE and Prior RL: Concentrations of anthocyanins in common foods in the United States and estimation of normal consumption. J Agric Food Chem 54: 4069-4075, 2006.

3. Wu X, Gu L, Prior RL and McKay S: Characterization of anthocyanins and proanthocyanidins in some cultivars of ribes, aronia, and sambucus and their antioxidant capacity. J Agric Food Chem 52: 7846-7856, 2004.

4. Pilaczynska-Szczesniak L, Skarpanska-Steinborn A, Deskur E, Basta P and Horoszkiewicz-Hassan M: The influence of chokeberry juice supplementation on the reduction of oxidative stress resulting from an incremental rowing ergometer exercise. Int $\mathbf{J}$ Sport Nutr Exerc Metab 15: 48-58, 2005.

5. Kähkönen MP, Hopia AI, Vuorela HJ, Rauha JP, Pihlaja K, Kujala TS and Heinonen M: Antioxidant activity of plant extracts containing phenolic compounds. J Agric Food Chem 47: 3954-3962, 1999.

6. Zheng W and Wang SY: Oxygen radical absorbing capacity of phenolics in blueberries, cranberries, chokeberries, and lingonberries. J Agric Food Chem 51: 502-509, 2003.

7. Rugină D, Sconţa Z, Leopold L, Pintea A, Bunea A and Socaciu C: Antioxidant activities of chokeberry extracts and the cytotoxic action of their anthocyanin fraction on HeLa human cervical tumor cells. J Med Food 15: 700-706, 2012.

8. Tjelle TE, Holtung L, Bøhn SK, Aaby K, Thoresen M, Wiik SA, Paur I, Karlsen AS, Retterst $\varnothing 1$ K, Iversen PO and Blomhoff R: Polyphenol-rich juices reduce blood pressure measures in a randomised controlled trial in high normal and hypertensive volunteers. Br J Nutr 114: 1054-1063, 2015.

9. Ciocoiu M, Badescu L, Miron A and Badescu M: The involvement of a polyphenol-rich extract of black chokeberry in oxidative stress on experimental arterial hypertension. Evid Based Complement Alternat Med 2013: 912769, 2013.

10. Sikora J, Broncel M, Markowicz M, Chałubiński M, Wojdan K and Mikiciuk-Olasik E: Short-term supplementation with Aronia melanocarpa extract improves platelet aggregation, clotting, and fibrinolysis in patients with metabolic syndrome. Eur J Nutr 51: 549-556, 2012. 
11. Mu J, Xin G, Zhang B, Wang Y, Ning C and Meng X: Beneficial effects of Aronia melanocarpa berry extract on hepatic insulin resistance in type 2 diabetes mellitus rats. J Food Sci 85: 1307-1318, 2020.

12. Takahashi A, Shimizu H, Okazaki Y, Sakaguchi H, Taira T, Suzuki T and Chiji H: Anthocyanin-rich phytochemicals from aronia fruits inhibit visceral fat Accumulation and hyperglycemia in high-fat diet-induced dietary obese rats. J Oleo Sci 64 1243-1250, 2015.

13. Kujawska M, Ignatowicz E, Ewertowska M, Oszmiański J and Jodynis-Liebert J: Protective effect of chokeberry on chemicalinduced oxidative stress in rat. Hum Exp Toxicol 30: 199-208, 2011.

14. Vagiri M and Jensen M: Influence of juice processing factors on quality of black chokeberry pomace as a future resource for colour extraction. Food Chem 217: 409-417, 2017.

15. Ohta T, Nashida-Hosotani Y, Hosokawa M and Miyashita K: The effects of ethanol extract from aronia juice-residue on lipid metabolism. Presented at the International Society for Nutraceuticals and Functional Foods (ISNFF), Sapporo, pp137, 2011.

16. Rask-Madsen $\mathrm{C}$ and Kahn CR: Tissue-specific insulin signaling, metabolic syndrome, and cardiovascular disease. Arterioscler Thromb Vasc Biol 32: 2052-2059, 2012.

17. Jonathan Q and Purnell JQ: Definitions, classification, and epidemiology of obesity. In: Endotext [Internet]. Feingold KR, Anawalt B, Boyce A, et al (eds). MDText.com, Inc., South Dartmouth, MA, 2000.

18. Kuwabara H, Takanami S, Oguri I, Yoshida T and Nakajima T: Influence of ascorbic acid on polyphenol determination in fruit juice. Res Rep Food Technol Res Inst Nagano Prefect 13: 49-53, 1985 (In Japanese).

19. Cassinese C, de Combarieu E, Falzoni M, Fuzzati N, Pace R and Sardone N: New liquid chromatography method with ultraviolet detection for analysis of anthocyanins and anthocyanidins in vaccinium myrtillus fruit dry extracts and commercial preparations. J AOAC Int 90: 911-919, 2007.

20. Reeves PG, Nielsen FH and Fahey GC Jr: AIN-93 purified diets for laboratory rodents: Final report of the American institute of nutrition ad hoc writing committee on the reformulation of the AIN-76A rodent diet. J Nutr 123: 1939-1951, 1993.

21. Folch J, Lees M and Sloane Stanley GH: A simple method for the isolation and purification of total lipides from animal tissues. J Biol Chem 226: 497-509, 1957.

22. Valcheva-Kuzmanova S, Kuzmanov K, Mihova V, Krasnaliev I, Borisova P and Belcheva A: Antihyperlipidemic effect of Aronia melanocarpa fruit juice in rats fed a high-cholesterol diet. Plant Foods Hum Nutr 62: 19-24, 2007.

23. Wawer I, Wolniak M and Paradowska K: Solid state NMR study of dietary fiber powders from aronia, bilberry, black currant and apple. Solid State Nucl Magn Reson 30: 106-113, 2006.

24. Hsu PK, Chien PJ, Chen CH and Chau CF: Carrot insoluble fiber-rich fraction lowers lipid and cholesterol absorption in hamsters. LWT-Food Sci Technol 39: 338-343, 2006.

25. Tanaka T and Tanaka A: Chemical components and characteristics of black chokeberry. J Jpn Soc Food Sci Technol 48: 606-610, 2001.
26. Shimano H, Yahagi N, Amemiya-Kudo M, Hasty AH, Osuga J, Tamura Y, Shionoiri F, Iizuka Y, Ohashi K, Harada K, et al: Sterol regulatory element-binding protein-1 as a key transcription factor for nutritional induction of lipogenic enzyme genes. J Biol Chem 274: 35832-35839, 1999.

27. Wang Z, Kim JH, Jang YS, Kim CH, Lee JY and Lim SS: Anti-obesity effect of Solidago virgaurea var. gigantea extract through regulation of adipogenesis and lipogenesis pathways in high-fat diet-induced obese mice (C57BL/6N). Food Nutr Res 61: $1273479,2017$.

28. Park H, Liu Y, Kim HS and Shin JH: Chokeberry attenuates the expression of genes related to de novo lipogenesis in the hepatocytes of mice with nonalcoholic fatty liver disease. Nutr Res 36: 57-64, 2016.

29. Rakhshandehroo M, Knoch B, Müller M and Kersten S: Peroxisome proliferator-activated receptor alpha target genes. PPAR Res 2010: 612089, 2010.

30. Nakatani T, Tsuboyama-Kasaoka N, Takahashi M, Miura S and Ezaki O: Mechanism for peroxisome proliferator-activated receptor-alpha activator-induced up-regulation of UCP2 mRNA in rodent hepatocytes. J Biol Chem 277: 9562-9569, 2002.

31. Birari RB and Bhutani KK: Pancreatic lipase inhibitors from natural sources: Unexplored potential. Drug Discov Today 12: 879-889, 2007.

32. Guo L, Gao Z, Zhang L, Guo F, Chen Y, Li Y and Huang C: Saponin-enriched sea cucumber extracts exhibit an antiobesity effect through inhibition of pancreatic lipase activity and upregulation of LXR- $\beta$ signaling. Pharm Biol 54: 1312-1325, 2016.

33. McDougall GJ, Kulkarni NN and Stewart D: Berry polyphenols inhibit pancreatic lipase activity in vitro. Food Chem 115: 193-199, 2009

34. Wang D, Xia M, Gao S, Li D, Zhang Y, Jin T and Ling W: Cyanidin-3-O- $\beta$-glucoside upregulates hepatic cholesterol $7 \alpha$-hydroxylase expression and reduces hypercholesterolemia in mice. Mol Nutr Food Res 56: 610-621, 2012.

35. Del Bas JM, Fernández-Larrea J, Blay M, Ardèvol A, Salvadó MJ, Arola L and Bladé C: Grape seed procyanidins improve atherosclerotic risk index and induce liver CYP7A1 and SHP expression in healthy rats. FASEB J 19: 479-481, 2005.

36. Momtazi AA, Banach M, Pirro M, Katsiki N and Sahebkar A: Regulation of PCSK9 by nutraceuticals. Pharmacol Res 120: 157-169, 2017.

37. Dávalos A, Fernández-Hernando C, Cerrato F, Martínez-Botas J, Gómez-Coronado D, Gómez-Cordovés C and Lasunción MA: Red grape juice polyphenols alter cholesterol homeostasis and increase LDL-receptor activity in human cells in vitro. J Nutr 136: 1766-1773, 2006.

This work is licensed under a Creative Commons Attribution-NonCommercial-NoDerivatives 4.0 International (CC BY-NC-ND 4.0) License. 